DOI: 10.12731/2070-7568-2020-4-313-327

УДК 658.58

\title{
МНОГОНОМЕНКЛАТУРНАЯ ОПТИМИЗАЦИЯ РАЗМЕРА ОБОРОТНОГО ФОНДА МЕТОДОМ РЕКУРСИИ ПРИ АГРЕГАТНОМ МЕТОДЕ РЕМОНТА
}

\section{Тюльпинова Н.В.}

Эффективное управление оборотным фондом запасных агрегатов требует наличия соответствующих программных решений. Таковые в настоящее время имеются, но основным сдерживающим фактором, препятствующим широкому внедрению этих инструментов в управленческую практику, является присущчий им существенный недостаток - традиционно низкий уровень вычислительной производительности и надежности. Причина - итеративное алгоритмическое ядро, которое, с одной стороны, обусловливает низкий уровень быстродействия вследствие большого объема избыточной вычислительной работьл, а с другой сторонь, порождает проблему «больших» чисел, приводямую как к некорректным результатам, так и к аварийным остановам расчетов из-за переполнения разрядной сетки. В процессе многономенклатурной оптимизации размера оборотного фонда обозначенные проблемы практически полностью парализуют вычислительный проиесс. В этой связи актуальной является разработка альтернативного решения, лишенного перечисленных недостатков. Для этого автором статьи предложено и разработано рекурсивное алгоритмическое ядро, полностью устраняющее все обозначенные дефекты имеющихся решений.

Цель - повышение уровня вычислительной производительности и надежности программных решений, предназначенных для управления оборотным фондом запасных агрегатов.

Метод или методология проведения работы: метод рекурсии и рекуррентных соотномений. 
Результаты: программно-алгоритмический инструментарий многономенклатурной оптимизации размера оборотного фонда методом рекурсии при агрегатном методе ремонта.

Область применения результатов: финансовый менеджмент.

Ключевые слова: многономенклатурная оптимизация; оборотный фонд; агрегатный метод ремонта; рекурсия.

\section{MULTI-ASSORTMENT OPTIMIZATION \\ OF REVERSIBLE FUND'S SIZE BASED ON RECURSION METHOD WITH THE AGGREGATE REPAIR METHOD}

\section{Tyulpinova N.V.}

Effective management of the reversible fund of spare parts requires appropriate software solutions. These are currently available, but the main constraint that prevents the widespread introduction of these tools into management practice is their inherent significant drawback - the traditionally low level of computing performance and reliability. The reason is an iterative algorithmic kernel, which, on the one hand, causes a low level of processing speed due to a large amount of redundant computational work, and on the other hand, generates a problem of «large» numbers, leading to both incorrect results and emergency stops of calculations because of overflow of the bit grid. During multi-assortment optimization of reversible fund's size, the indicated problems almost completely paralyze the computational process. In this regard, the development of an alternative solution, devoid of the listed disadvantages, is relevant. For this, the author of the article proposed and developed a recursive algorithmic kernel that completely eliminates all the indicated defects of the existing solutions.

Purpose - increasing the computing performance and reliability of software solutions for management by the reversible fund of spare parts.

Method or methodology of the work: recursion and recurrence method.

Results: software and algorithms for multi-assortment optimization of reversible fund's size based on recursion method with the aggregate repair method. 


\section{Practical implications: financial management.}

Keywords: multi-assortment optimization; reversible fund; aggregate repair method; recursion.

Исследование различных аспектов деятельности автотранспортных предприятий [1-15] показывает, что одной из наиболее важных задач для этих предприятий является эффективное управление оборотным фондом запасных агрегатов, узлов, приборов и деталей, так как, с одной стороны, при недостаточном размере оборотного фонда не представляется возможным использование главного преимущества агрегатного метода ремонта - повышения коэффициента технической готовности автопарка и решения задачи сокращения простоев автомобилей в зоне текущего ремонта, а, с другой стороны, при завышенном размере оборотного фонда затраты на приобретение и хранение чрезмерного складского запаса значительно возрастают и оказываются выше потерь, связанных с простоем автомобилей из-за нехватки исправных агрегатов оборотного фонда. Поэтому для принятия соответствующего управленческого решения необходима предварительная количественная оценка, требующая разработки и реализации такой оптимизационной модели, которая методом последовательного сканирования на компьютере определит искомый оптимум размера оборотного фонда, обеспечивающий минимум целевой функции суммарных затрат.

Традиционная методика определения суммарных затрат при агрегатном методе ремонта [1-15] базируется на математическом аппарате теории массового обслуживания и, в общем случае, сводится к расчету последовательности из семи параметров (рис. 1a): 1) $\mathrm{P}_{\text {free }}$ вероятность того, что все обслуживающие агрегаты свободны; 2) $\mathrm{P}_{\text {job }}$ - вероятность того, что все обслуживающие агрегаты заняты; 3) $\mathrm{A}_{\mathrm{dev}}$ - среднее число неисправных автомобилей в накопителе; 4) $\mathrm{A}_{\text {job }}$ - среднее число свободных обслуживающих агрегатов; 5) Z1 затраты, вызванные простоем автомобилей в ожидании поступления отремонтированных агрегатов; 6) Z2 - затраты, вызванные неиспользованием отремонтированных агрегатов; 7) Z - суммарные затраты. Исходными данными для такого расчета являются: 1) $\lambda$ - 
средняя интенсивность поступления автомобилей в ремонт, агр./сут.; 2) Т - среднее время оборота (возврата) агрегата на склад, сут.; 3) C1 - издержки от простоя одного автомобиля, у.е.; 4) С2 - издержки от неиспользования одного отремонтированного агрегата, у.е.

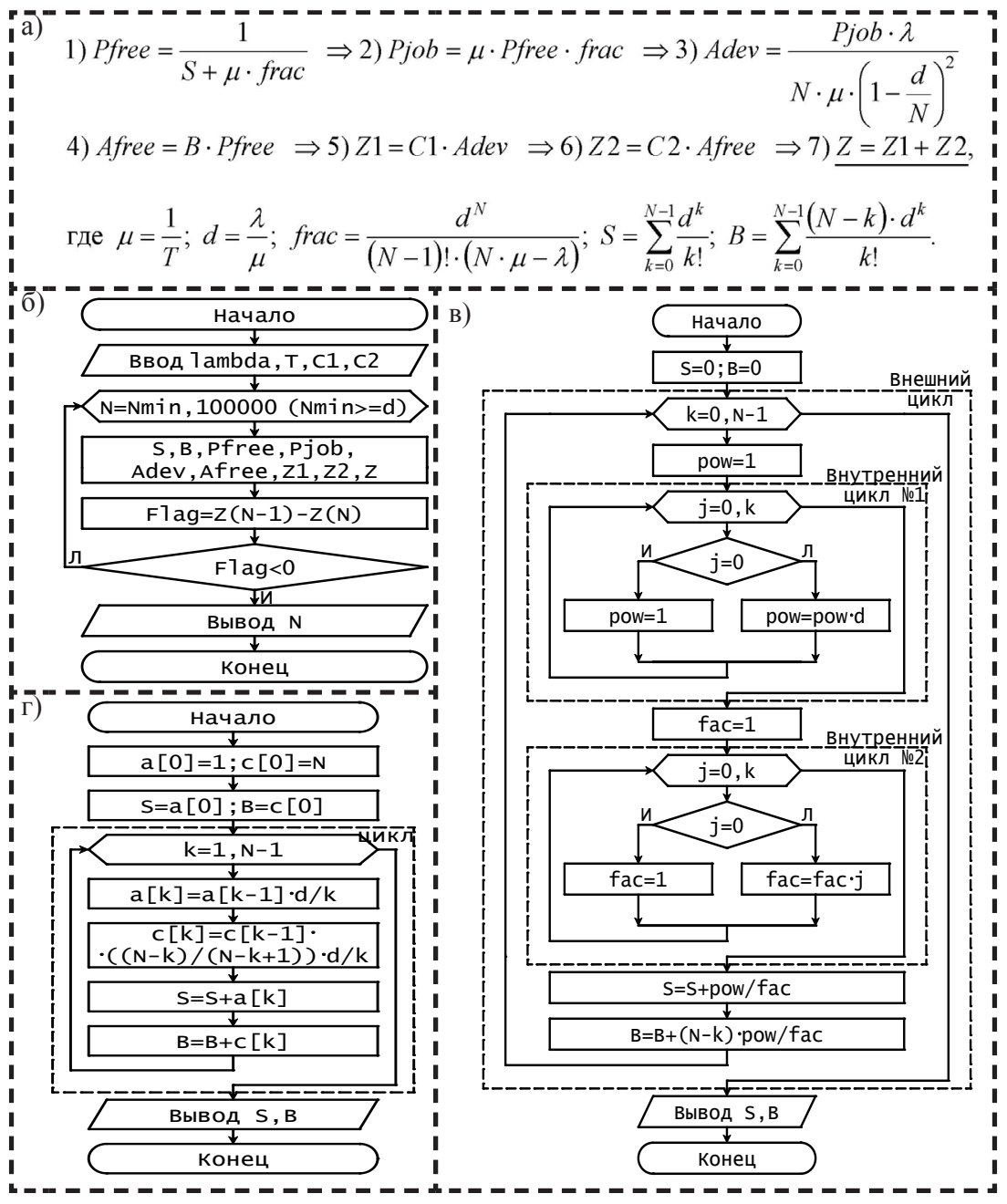

Рис. 1. Алгоритм оптимизации при традиционном и рекурсивном подходе 
Для решения задачи оптимизации целевой функции суммарных затрат Z необходимо организовать многократно повторяющийся, циклический расчет (рис. 1б) величины Z для серии значений $\mathrm{N}$, последовательно увеличивающихся от минимально необходимой величины Nmin до искомого оптимума, при котором значение Z достигает своего минимума, а критерием прерывания цикла и завершения расчета при этом может служить разница Z при двух последовательных N (переменная flag на блок-схеме).

На каждой итерации цикла оптимизации необходимо произвести расчёт двух, входящих в Z, вспомогательных величин $\mathrm{S}$ и $\mathrm{B}$, каждая из которых представляет собой сумму ряда, эффективность вычисления которой в значительной степени определяет эффективность оптимизации. Однако в традиционной методике определения $\mathrm{Z}$ данному аспекту (а именно, расчету S и B) в настоящее время не уделяется должного внимания: обзор существующих программноалгоритмических моделей [1-15], реализующих прогнозирование потребности в запасных частях, показывает, что все они базируются на стандартном подходе и ограничиваются программированием суммы ряда, основываясь лишь на готовой формуле общего члена ряда, что в итоге приводит к крайне низкой эффективности получаемого в этом случае программного обеспечения по следующим причинам:

- во-первых, такой подход характеризуется низким уровнем быстродействия, так как требует выполнения большого объема избыточной вычислительной работы, реализация которой на компьютере требует существенных затрат процессорного времени, что особенно чувствительно в случае многономенклатурной оптимизации, охватывающей не одно наименование оборотных агрегатов, а спектр из десятков и сотен наименований изделий. Причина в том, что внутри одного внешнего (основного) цикла по накоплению суммы необходимо организовать дополнительно ещё два внутренних (вспомогательных) цикла по накоплению двух произведений, содержащихся в числителе и знаменателе величин $\mathrm{S}$ и $\mathrm{B}$ (рис. 1в): цикл №1 - для накопления степени числителя, цикл №2 - для накопления факториала знаменателя; 
- во-вторых, не гарантируется корректность конечного результата, поскольку при организации отдельных циклов для накопления степени и факториала можно получить «большие» числа, при делении которых друг на друга произойдет потеря точности, так как количество значащих цифр, хранимых в ячейке памяти, ограничено;

- в-третьих, не учитывается возможность переполнения разрядной сетки, обусловленная появлением «больших» чисел, которые не помещаются в имеющемся количестве разрядов вычислительного устройства, что является основной и наиболее частой причиной аварийных остановов большинства существующих программных приложений, реализующих данный подход. В этой связи весьма актуальной становится задачи разработки программно-алгоритмического инструментария, лишенного перечисленных недостатков.

Для решения поставленной задачи автором статьи предлагается альтернативный подход (рис. 1г), базирующийся на обнаружении и использовании в $\mathrm{S}$ и В рекуррентной связи между слагаемыми, которая позволит обойтись одним эффективным простым циклом, вместо трех неэффективных вложенных циклов традиционного подхода, другими словами, метод рекурсии открывает возможность существенного повышения эффективности процедуры вычислительной оптимизации в целом.

Математические выкладки, описывающие аналитический вывод рекуррентных формул и коэффициентов рекурсии для $\mathrm{S}$ и $\mathrm{B}$ представлены на рис. 2: полученные формулы позволяют вычислять значение очередного члена на основе значения предыдущего члена ряда. Сопоставление общей формулы члена ряда и рекуррентной показывает, что рекуррентная формула значительно упрощает вычисления, так как лишена и степени, и факториала, а, следовательно, циклы для их расчета не нужны. В итоге остаётся один единственный цикл для накопления $\mathrm{S}$ и $\mathrm{B}$, который, благодаря отсутствию факториалов и степеней, не вызывает появления «больших» чисел, а поэтому, проблемы обеспечения точности вычисления и переполнения разрядной сетки снимаются автоматически. 


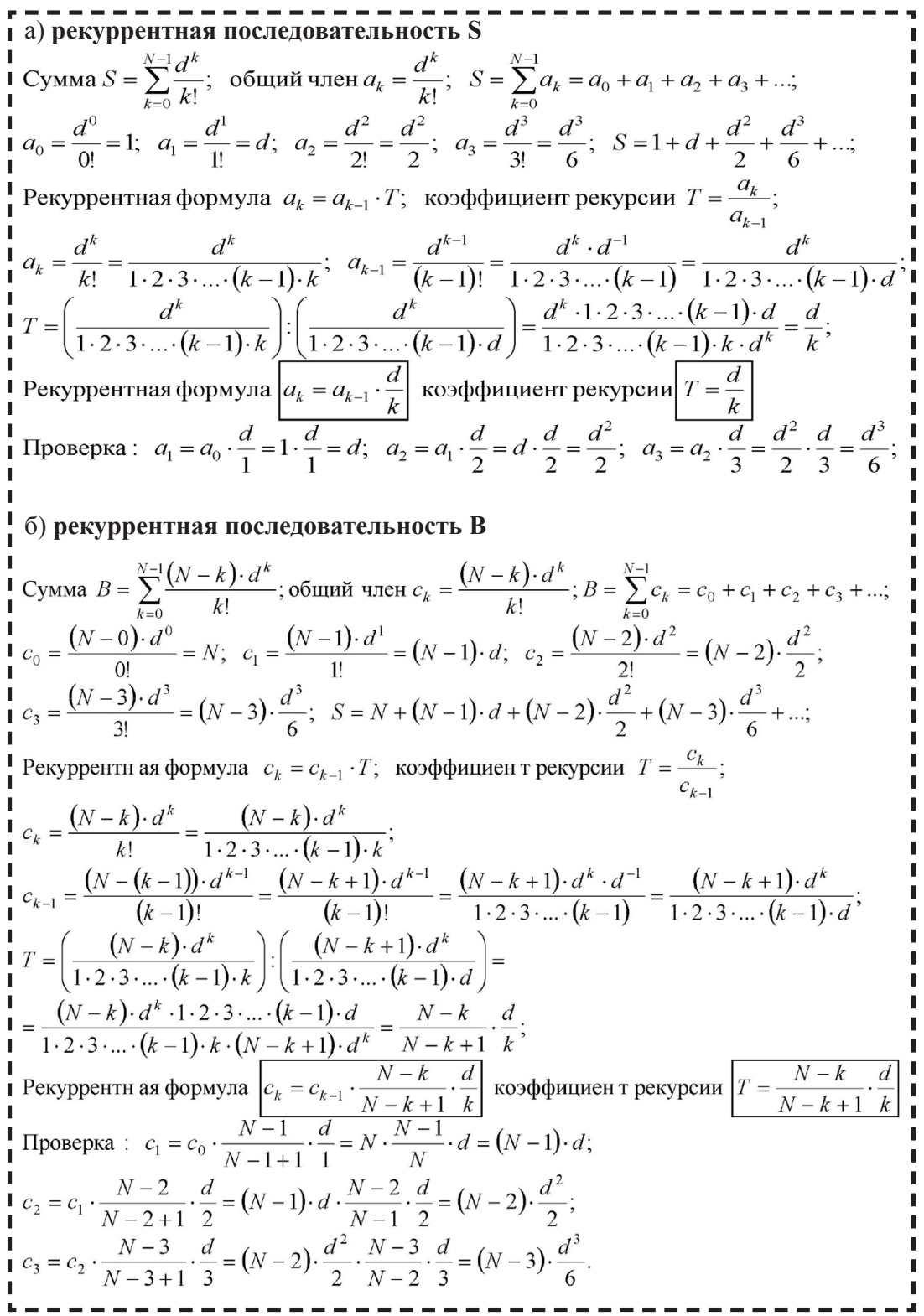

Рис. 2. Рекуррентные формулы и коэффициенты рекурсии 
Для наглядности сопоставления предлагаемой и традиционной методик на рис. 3 приведены вычислительные протоколы для одного значения $\mathrm{N}=7$ (расчет произведен при $\mathrm{d}=6$ ): видно, что рекурсия существенно сокращает объем вычислений. А поскольку в процессе оптимизации требуется расчет не для одного $\mathrm{N}$, а для серии последовательно увеличивающихся $\mathrm{N}$ (т.е. для $\mathrm{N}=7, \mathrm{~N}=8$,

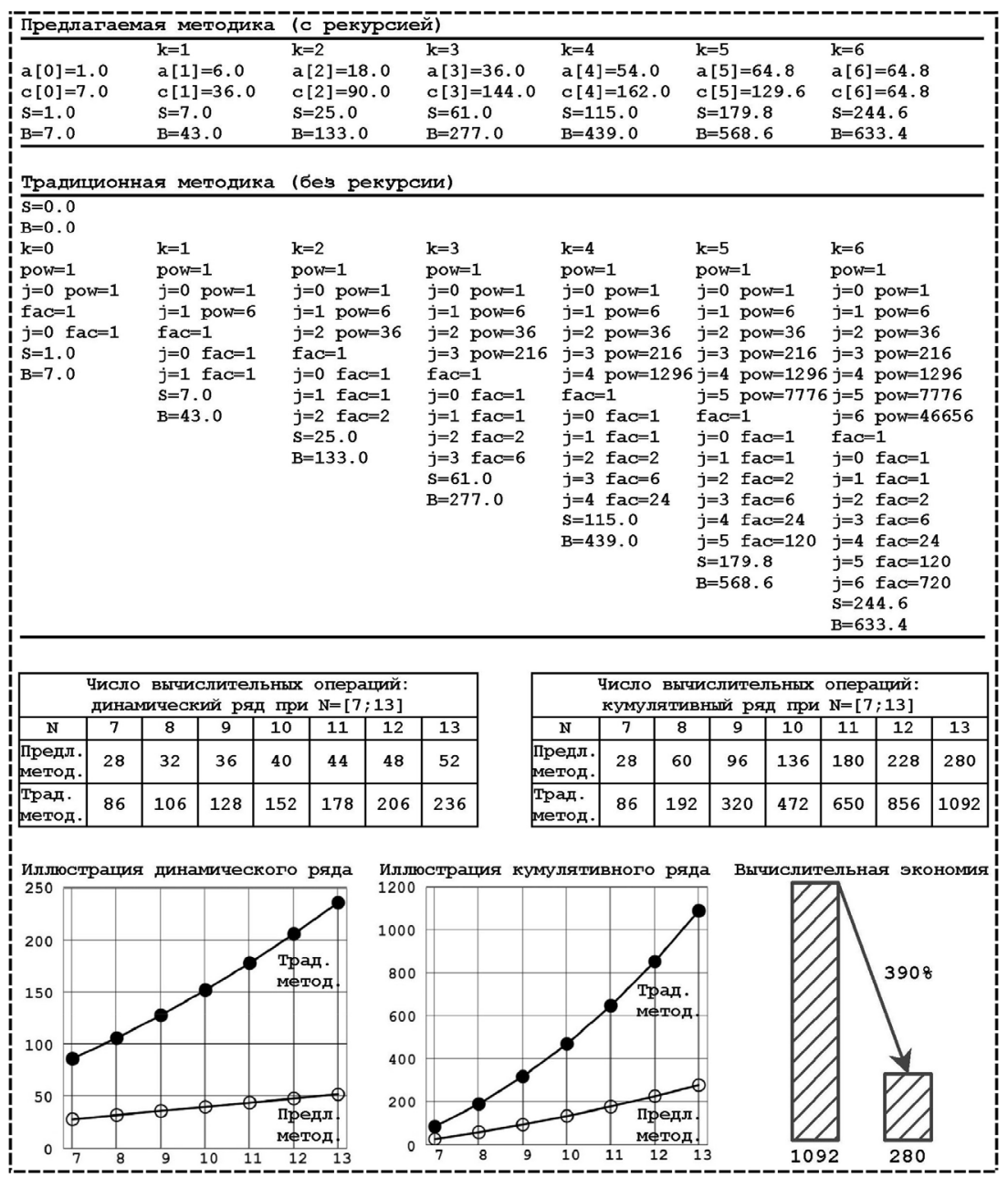

Рис. 3. Число вычислительных операций по двум методикам 
$\mathrm{N}=9$ и т.д. до тех пор, пока не будет достигнут минимум суммарных затрат, например, до $\mathrm{N}=13$ ), то на рис. 3 также представлено сравнение требуемого в этом случае общего объема вычислительной работы для всех значений $\mathrm{N}$ при помощи динамических и кумулятивных рядов и иллюстрирующих их графиков. Сопоставление показывает, что в данном конкретном случае применение предлагаемой методики (с рекурсией) существенно - на 390\% (или приблизительно в 4 раза) - снижает число вычислительных операций по сравнению с традиционной методикой (без рекурсии).

Следует заметить, что на рис. 3 приведено сопоставление лишь для одного наименования номенклатуры автопарка, а при многономенклатурной оптимизации (десятки и сотни наименований) сокращение объема вычислений окажется весьма ощутимее (по 390\% с каждого отдельного наименования). А с ростом $\mathrm{N}$ вычислительная экономия возрастает вообще в геометрической прогрессии по причине быстрого роста факториалов в традиционной методике.

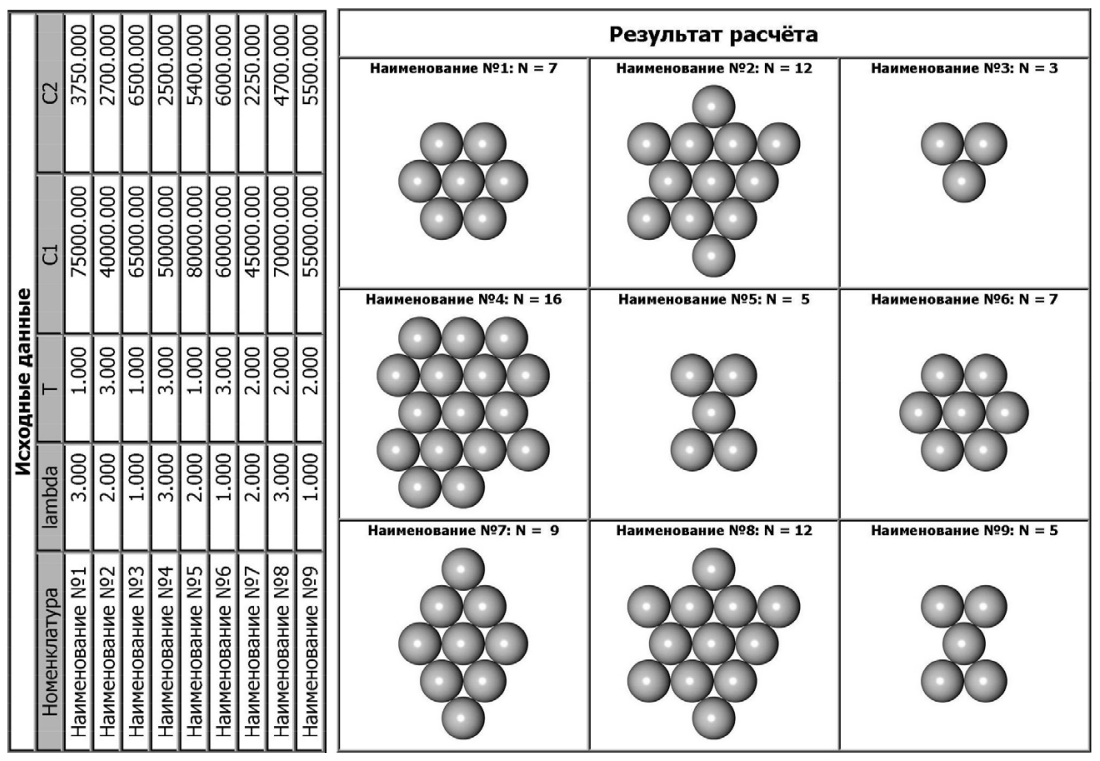

Рис. 4. Пример работы разработанного программного обеспечения (начало) 


\begin{tabular}{|c|c|c|c|c|c|c|c|c|}
\hline \multicolumn{9}{|c|}{ Протоколрасчёта } \\
\hline \multicolumn{9}{|c|}{ Наименование №1 } \\
\hline $\mathrm{N}$ & $\mathrm{S}$ & \multicolumn{2}{|c|}{ B } & Pfree & Pjob & \multicolumn{2}{|c|}{ Adev } & Afree \\
\hline 4 & 13.000000 & \multicolumn{2}{|c|}{26.500000} & 0.037736 & 0.509434 & \multicolumn{2}{|c|}{6.113208} & 1.000000 \\
\hline 5 & 16.375000 & \multicolumn{2}{|c|}{42.875000} & 0.046647 & 0.236152 & \multicolumn{2}{|c|}{0.885569} & 2.000000 \\
\hline 6 & 18.400000 & \multicolumn{2}{|c|}{61.275000} & 0.048960 & 0.099143 & \multicolumn{2}{|c|}{0.198286} & 3.000000 \\
\hline 7 & 19.412500 & \multicolumn{2}{|c|}{80.687500} & 0.049574 & 0.037645 & \multicolumn{2}{|c|}{0.049409} & 4.000000 \\
\hline 8 & 19.846429 & \multicolumn{2}{|c|}{100.533929} & 0.049734 & 0.012949 & \multicolumn{2}{|c|}{0.012431} & 5.000000 \\
\hline $\mathrm{N}$ & \multicolumn{2}{|l|}{$\mathrm{Z1}$} & & $\mathrm{Z2}$ & $Z$ & & & Flag \\
\hline 4 & 458490 & 566038 & & 3750.000000 & 462240 & & & $=--$ \\
\hline 5 & 66417 & 538484 & & 7500.000000 & 73917 & 484 & & 388322.927554 \\
\hline 6 & 14871 & 181028 & & 11250.000000 & 26121 & $\overline{028}$ & & 47796.157456 \\
\hline 7 & 3705 & 702943 & & 15000.000000 & 18705 & 943 & & 7415.778085 \\
\hline 8 & 932 & 307856 & & 18750.000000 & 19682 & 856 & & -976.604913 \\
\hline & & & & Іаименовани & & & & \\
\hline $\mathrm{N}$ & $\bar{S}$ & $\bar{B}$ & & Pfree & Pjob & $\overline{\mathrm{Ac}}$ & & Afree \\
\hline 7 & 244.600000 & 633. & 400000 & 0.001579 & 0.613830 & & 780865 & 1.000000 \\
\hline 8 & 300.142857 & 933. & 542857 & 0.002142 & 0.356981 & & 283773 & 2.000000 \\
\hline 9 & 341.800000 & 1275 . & 342857 & 0.002352 & 0.195981 & & 175885 & 3.000000 \\
\hline 10 & 369.571429 & 1644. & 914286 & 0.002432 & 0.101299 & & 379872 & 4.000000 \\
\hline 11 & 386.234286 & 2031. & 148571 & 0.002462 & 0.049222 & & 129946 & 5.000000 \\
\hline 12 & 395.323117 & 2426. & 471688 & 0.002473 & 0.022474 & & 044948 & 6.000000 \\
\hline 13 & 399.867532 & 2826 . & 339221 & 0.002477 & 0.009647 & & 015357 & 7.000000 \\
\hline $\bar{N}$ & $\mathrm{Z1}$ & & & 22 & $\mathrm{Z}$ & & & Flag \\
\hline 7 & 1031234 & 606883 & & 2700.000000 & 1033934 & 383 & & ---- \\
\hline 8 & 171350 & 921222 & & 5400.000000 & 176750 & 222 & & 857183.685662 \\
\hline 9 & 47035 & 419047 & & 8100.000000 & 55135 & 047 & & 121615.502175 \\
\hline 10 & 15194 & 886403 & & 10800.000000 & 25994 & 403 & & 29140.532644 \\
\hline 11 & 5197 & 840490 & & 13500.000000 & 18697 & 490 & & 7297.045913 \\
\hline 12 & 1797 & 935242 & & 16200.000000 & 17997 & 242 & & 699.905248 \\
\hline 13 & 614 & 275961 & & 18900.000000 & 19514 & 961 & & -1516.340719 \\
\hline & & & & Іаименовани & & & & \\
\hline $\mathrm{N}$ & $\mathrm{S}$ & $\bar{B}$ & & Pfree & Pjob & $\overrightarrow{A C}$ & & Afree \\
\hline 2 & 2.000000 & & 000000 & 0.333333 & 0.333333 & & 666667 & 1.000000 \\
\hline 3 & 2.500000 & & 500000 & 0.363636 & 0.090909 & & 068182 & 2.000000 \\
\hline 4 & 2.666667 & & 166667 & 0.367347 & 0.020408 & & 009070 & 3.000000 \\
\hline $\mathrm{N}$ & $\overline{\mathrm{Z1}}$ & & & $\mathrm{Z2}$ & $\bar{z}$ & & & Flag \\
\hline 2 & 43333 & 333333 & & 6500.000000 & 49833 & 333 & & 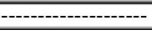 \\
\hline 3 & 4431 & 818182 & & 13000.000000 & 17431 & 182 & & 32401.515152 \\
\hline 4 & 589 & 569161 & & 19500.000000 & 20089 & 161 & & -2657.750979 \\
\hline & & & & Іаименовани & & & & \\
\hline $\mathrm{N}$ & $\mathrm{S}$ & $\bar{B}$ & & Pfree & Pjob & $\overline{\mathrm{AC}}$ & & Afree \\
\hline 10 & 4759.818304 & 14368. & 461384 & 0.000070 & 0.668732 & & 185837 & 1.000000 \\
\hline 11 & 5720.682612 & 20089. & 143996 & 0.000100 & 0.430470 & & 654139 & 2.000000 \\
\hline 12 & 6506.844318 & 26595. & 988314 & 0.000113 & 0.266035 & & 192416 & 3.000000 \\
\hline 13 & 7096.465598 & 33692. & 453912 & 0.000119 & 0.157501 & & 151725 & 4.000000 \\
\hline 14 & 7504.664946 & 41197. & 118858 & 0.000121 & 0.089176 & & 449447 & 5.000000 \\
\hline 15 & 7767.078812 & 48964. & 197670 & 0.000123 & 0.048234 & & 180876 & 6.000000 \\
\hline 16 & 7924.527132 & 56888. & 724802 & 0.000123 & 0.024909 & & 073202 & 7.000000 \\
\hline 17 & 8013.091812 & 64901. & 816613 & 0.000123 & 0.012281 & & 029360 & 8.000000 \\
\hline $\mathrm{N}$ & $\mathrm{Z1}$ & & & $\mathrm{Z2}$ & Z & & & Flag \\
\hline 10 & 3009291 & 858485 & & 2500.000000 & 3011791. & 485 & - & - \\
\hline 11 & 532706 & 930326 & & 5000.000000 & 537706 & 326 & & 2474084.928158 \\
\hline 12 & 159620 & 810681 & & 7500.000000 & 167120 & 681 & & 370586.119645 \\
\hline 13 & 57586 & 263362 & & 10000.000000 & 67586 & 362 & & 99534.547319 \\
\hline 14 & 22472 & 351129 & & 12500.000000 & 34972 & 129 & & 32613.912233 \\
\hline 15 & 9043 & 820190 & & 15000.000000 & 24043 & 190 & & 10928.530939 \\
\hline 16 & 3660 & 081512 & & 17500.000000 & 21160 & 512 & & 2883.738678 \\
\hline 17 & 1468 & 005474 & & 20000.000000 & 21468 & & & -307.923962 \\
\hline
\end{tabular}

Рис. 4. Пример работы разработанного программного обеспечения (окончание) 
Представленное сравнение результативности традиционного итеративного и предлагаемого рекурсивного алгоритмов убедительно доказывает, что именно рекурсия и полученные на ее основе рекуррентные соотношения позволяют достичь поставленной цели и существенно повысить уровень вычислительной производительности и надежности получаемых программных решений. На базе описанного рекурсивного алгоритма сгенерирован программный код, положенный в основу разработанного автором статьи программного средства многономенклатурной оптимизации (рис. 4): исходные данные задаются в табличном формате, а результаты расчета выводятся в наглядной графической и табличной числовой форме, содержащей протокол расчета по каждому наименованию.

Разработанное программное обеспечение и его рекурсивное алгоритмическое ядро представляет собой надежный и высокоскоростной инструмент многономенклатурной оптимизации для поддержки принятия обоснованных решений в сфере управления оборотным фондом запасных агрегатов, а предложенный алгоритм дает возможность полностью устранить типичные недостатки, присущие имеющимся в данной области решениям, обеспечивая: 1) существенное увеличение быстродействия вычислительной процедуры оптимизации; 2) гарантированное получение корректного конечного результата; 3) отсутствие аварийных остановов, вызванных переполнением разрядной сетки.

\section{Список литературы}

1. Верительник Е.А., Панайотов К.К., Таращанский М.Т. Определение количества хранимых запасных частей на автопредприятиях с учетом ограниченности оборотных средств // Вестник Луганского национального университета имени Владимира Даля. 2019. №1 (19). C. 30-34.

2. Воронина И.Ф., Судак Ф.М., Негурица В.В., Веденичев А.И. Прогнозирование потребности в запасных частях для автотранспортных предприятий // Вести Автомобильно-дорожного института. 2020. №3 (34). C. 33-37. 
3. Джолабов Ю.Ш., Карданов Х.Б. Агрегатный метод ремонта: перспективы его применения в современном ремонтном производстве // Известия Кабардино-Балкарского аграрного университета им. В.М. Кокова. 2020. №1 (27). С. 93-97.

4. Егоров С.Я., Салих Х.С., Затонский А.В. Имитационная модель технической готовности крупного автопарка // Вестник Южно-Уральского государственного университета. Серия: Компьютерные технологии, управление, радиоэлектроника. 2020. Т.20. №2. С. 14-25.

5. Кабикенов С.Ж., Кызылбаева Э.Ж., Лажимова А.Б. Анализ методик расчета запасных частей автомобилей // Вестник Казахской академии транспорта и коммуникаций им. М. Тынышпаева. 2017. №2 (101). C. 79-83.

6. Кызылбаева Э.Ж., Кадыров А.С. Анализ математических моделей прогнозирования запасных частей // Наука и техника Казахстана. 2018. №2. С. 56-62.

7. Судак Ф.М., Воронина И.Ф., Еремин А.В., Новиков Г.В. Оптимизация затрат на управление резервом запасных частей на автосервисных предприятиях // Вести Автомобильно-дорожного института. 2019. №2 (29). С. 35-41.

8. Судак Ф.М., Воронина И.Ф., Заика А.И. Усовершенствование методики расчета необходимого количества запасных частей на предприятиях автомобильного транспорта // Вести Автомобильно-дорожного института. 2018. №3 (26). С. 44-48.

9. Тахтамышев Х.М., Колодницкая О.А. Оптимизация оборотного фонда узлов и агрегатов автомобилей на АТП при различных системах снабжения // Научный вестник ГАОУ ВПО «Невиномысский государственный гуманитарно-технический институт». 2016. №4. С. 44-48.

10. Терловая В.И. Теоретические и практические аспекты управления затратами автотранспортного предприятия // Экономика строительства и природопользования. 2018. №4 (69). С. 108-115.

11. Block J., Ahmadi A., Xiao X., Kumar U. Spares provisioning strategy for periodically replaced units within the fleet retirement period // International Journal of System Assurance Engineering and Management. 2019. Vol. 10, pp. 299-315. 
12. Duan L., Xu B., Cao J., Wang X. Model of spare parts optimization based on system supply availability // Advances in Engineering Research. 2017. Vol. 124, pp. 386-392.

13. Duran O., Afonso P.S., Duran P.A. Spare parts cost management for long-term economic sustainability: using fuzzy activity based LCC // Sustainability. 2019. Vol. 11. No. 7, pp. 1-14.

14.Pan G., Luo Q., Li X., Wang Y., Huang C. Model of spare parts optimization based on GA for equipment // Advances in Intelligent Systems Research. 2018. Vol. 160, pp. 44-47.

15. Wang H., Li D., Yi H. Optimal allocation of fleet's spare parts for system reliability // Journal of Shanghai Jiaotong University (Science). 2017. Vol. 22. No. 6, pp. 664-671.

\section{References}

1. Veritel'nik E.A., Panayotov K.K., Tarashchanskiy M.T. Opredelenie kolichestva khranimykh zapasnykh chastey na avtopredpriyatiyakh $\mathrm{s}$ uchetom ogranichennosti oborotnykh sredstv [Determination of the amount of stored spare parts for auto enterprises, considering the limited working capital]. Vestnik Luganskogo natsional'nogo universiteta imeni Vladimira Dalya. 2019. No. 1 (19), pp. 30-34.

2. Voronina I.F., Sudak F.M., Neguritsa V.V., Vedenichev A.I. Prognozirovanie potrebnosti $\mathrm{v}$ zapasnykh chastyakh dlya avtotransportnykh predpriyatiy [Forecasting of the spare parts need for motor transport companies]. Vesti Avtomobil'no-dorozhnogo instituta. 2020. No. 3 (34), pp. 33-37.

3. Dzholabov Yu.Sh., Kardanov Kh.B. Agregatnyy metod remonta: perspektivy ego primeneniya v sovremennom remontnom proizvodstve [Aggregate repair method: prospects of it's applications in modern repair production]. Izvestiya Kabardino-Balkarskogo agrarnogo universiteta im. V.M. Kokova. 2020. No. 1 (27), pp. 93-97.

4. Egorov S.Ya., Salikh Kh.S., Zatonskiy A.V. Imitatsionnaya model' tekhnicheskoy gotovnosti krupnogo avtoparka [Queue model of technical readiness of large car fleet]. Vestnik Yuzhno-Ural'skogo gosudarstvennogo universiteta. Seriya: Komp'yuternye tekhnologii, upravlenie, radioelektronika. 2020. Vol. 20. No. 2, pp. 14-25. 
5. Kabikenov S.Zh., Kyzylbaeva E.Zh., Lazhimova A.B. Analiz metodik rascheta zapasnykh chastey avtomobiley [Analysis of calculation methods of automotive spare parts]. Vestnik Kazakhskoy akademii transporta i kommunikatsiy im. M. Tynyshpaeva. 2017. No. 2 (101), pp. 79-83.

6. Kyzylbaeva E.Zh., Kadyrov A.S. Analiz matematicheskikh modeley prognozirovaniya zapasnykh chastey [Analysis of the mathematical models of forecasting spare parts]. Nauka i tekhnika Kazakhstana. 2018. No. 2, pp. 56-62.

7. Sudak F.M., Voronina I.F., Eremin A.V., Novikov G.V. Optimizatsiya zatrat na upravlenie rezervom zapasnykh chastey na avtoservisnykh predpriyatiyakh [Cost optimization to manage spare parts reserve at service centers]. Vesti Avtomobil'no-dorozhnogo instituta. 2019. No. 2 (29), pp. 35-41.

8. Sudak F.M., Voronina I.F., Zaika A.I. Usovershenstvovanie metodiki rascheta neobkhodimogo kolichestva zapasnykh chastey na predpriyatiyakh avtomobil'nogo transporta [Design procedure improvement of necessary number of spare parts at automobile transport enterprises]. Vesti Avtomobil'no-dorozhnogo instituta. 2018. No. 3 (26), pp. 44-48.

9. Takhtamyshev Kh.M., Kolodnitskaya O.A. Optimizatsiya oborotnogo fonda uzlov i agregatov avtomobiley na ATP pri razlichnykh sistemakh snabzheniya [Optimization of the working capital fund components and assemblies car trucking companies in different supply systems]. Nauchnyy vestnik GAOU VPO «Nevinomysskiy gosudarstvennyy gumanitarno-tekhnicheskiy institut». 2016. No. 4, pp. 44-48.

10. Terlovaya V.I. Teoreticheskie i prakticheskie aspekty upravleniya zatratami avtotransportnogo predpriyatiya [Theoretical and practical aspects of cost management of a motor transport enterprise]. Ekonomika stroitel'stva i prirodopol'zovaniya. 2018. No. 4 (69), pp. 108-115.

11. Block J., Ahmadi A., Xiao X., Kumar U. Spares provisioning strategy for periodically replaced units within the fleet retirement period. International Journal of System Assurance Engineering and Management. 2019. Vol. 10, pp. 299-315.

12. Duan L., Xu B., Cao J., Wang X. Model of spare parts optimization based on system supply availability. Advances in Engineering Research. 2017. Vol. 124, pp. 386-392. 
13. Duran O., Afonso P.S., Duran P.A. Spare parts cost management for long-term economic sustainability: using fuzzy activity based LCC. Sustainability. 2019. Vol. 11. No. 7, pp. 1-14.

14.Pan G., Luo Q., Li X., Wang Y., Huang C. Model of spare parts optimization based on GA for equipment. Advances in Intelligent Systems Research. 2018. Vol. 160, pp. 44-47.

15. Wang H., Li D., Yi H. Optimal allocation of fleet's spare parts for system reliability. Journal of Shanghai Jiaotong University (Science). 2017. Vol. 22. No. 6, pp. 664-671.

\section{ДАННЫЕ ОБ АВТОРЕ}

Тюльпинова Нина Владимировна, доцент кафедры «Технология машиностроения», кандидат технических наук, доцент Брянский государственный технический университет бульвар 50 лет Октября, 7, г. Брянск, 241035, Российская Федерация ninatulpinova@mail.ru

\section{DATA ABOUT THE AUTHOR}

Tyulpinova Nina Vladimirovna, Associate Professor «Manufacturing engineering», Candidate of Engineering Sciences, Associate Professor

Bryansk State Technical University

7, 50 let Octyabrya Blvd, Bryansk, 241035, Russian Federation ninatulpinova@mail.ru

ORCID: 0000-0002-1485-7 